\title{
Article
}

\section{Secular Trends in Ablation Therapy for Graves' Disease: An Analysis of a 15-Year Experience at a Tertiary Hospital in South Korea}

\author{
Min Joo Kim ${ }^{1,2,3,+}{ }^{1}$, Ye An Kim ${ }^{1,2,4,+}{ }^{1}$, Sun Wook Cho ${ }^{1,2, *} \mathbb{C}$, Su-jin Kim ${ }^{5}$, Kyu Eun Lee ${ }^{5}$, Young Joo Park ${ }^{1,2} \mathbb{D}^{\text {, }}$ \\ Do Joon Park ${ }^{1,2}$ and Bo Youn Cho ${ }^{1,2,6}$
}

1 Department of Internal Medicine, Seoul National University Hospital, Seoul 03080, Korea; chorong24@gmail.com (M.J.K.); yeanin@gmail.com (Y.A.K.); yjparkmd@snu.ac.kr (Y.J.P.); djpark@snu.ac.kr (D.J.P.); bycho@cau.ac.kr (B.Y.C.)

2 Department of Internal Medicine, Seoul National University College of Medicine, Seoul 03080, Korea

3 Seoul National University Hospital Healthcare System Gangnam Center, Seoul 06236, Korea

4 Department of Internal Medicine, Veterans Health Service Medical Center, Seoul 05368, Korea

5 Department of Surgery, Seoul National University Hospital, Seoul 03080, Korea; su.jin.kim.md@gmail.com (S.j.K.); kyu.eun.lee.md@gmail.com (K.E.L.)

6 Thyroid Center, Chung-Ang University Hospital, Seoul 06973, Korea

* Correspondence: swchomd@snu.ac.kr; Tel.: +82-2-2072-4761

+ These authors contributed equally to this work.

Citation: Kim, M.J.; Kim, Y.A.; Cho, S.W.; Kim, S.j.; Lee, K.E.; Park, Y.J.; Park, D.J.; Cho, B.Y. Secular Trends in Ablation Therapy for Graves' Disease: An Analysis of a 15-Year Experience at a Tertiary Hospital in South Korea. J. Clin. Med. 2021, 10, 1629.

https://doi.org/10.3390/jcm10081629

Academic Editor: Andrzej Lewinski

Received: 26 February 2021

Accepted: 9 April 2021

Published: 12 April 2021

Publisher's Note: MDPI stays neutral with regard to jurisdictional claims in published maps and institutional affiliations.

Copyright: (c) 2021 by the authors. Licensee MDPI, Basel, Switzerland. This article is an open access article distributed under the terms and conditions of the Creative Commons Attribution (CC BY) license (https:// creativecommons.org/licenses/by/ $4.0 /)$.

\begin{abstract}
Ablation therapy, such as radioactive iodine (RAI) therapy or thyroidectomy, is generally used as the second-line treatment for Graves' disease (GD) in Asia. This study investigated changes in the clinical characteristics and outcomes of ablation therapies for GD over 15 years. Patients who underwent ablation therapy between 2001 and 2015 at a single tertiary hospital were included. Among the 10,991 GD patients treated over this 15-year period, 1357 (12.3\%) underwent ablation therapy, and the most common reason was intractable GD. The proportion of patients who underwent any type of ablation therapy significantly decreased from $9.0 \%$ (2001-2005) to $7.7 \%$ (2011-2015). However, the proportion of patients who underwent surgery significantly increased from $1.1 \%$ (2001-2005) to 2.4\% (2011-2015), and the proportion of patients who received ablation therapy due to suspected thyroid cancer increased from $5 \%$ to $13 \%$ over time. With a median follow-up duration of 6.2 years, remission was achieved in $86 \%$ and $98 \%$ of patients in the RAI and surgery groups, respectively, and these rates remained stable over time. In conclusion, although the proportion of patients who underwent ablation therapy for GD decreased during 15 years, the proportion of those who underwent surgery increased in association with the increased rate of suspected thyroid cancers.
\end{abstract}

Keywords: ablation therapy; Graves' disease; hyperthyroidism; radioactive iodine (RAI); surgery

\section{Introduction}

The therapeutic options for Graves' disease (GD) include antithyroid drug (ATD) therapy, radioactive iodine (RAI) therapy using ${ }^{131} \mathrm{I}$, and thyroidectomy. Although several guidelines recommend the optimal indications for each treatment [1,2], the first-line treatment of choice for GD shows regional differences according to patients' or doctors' preferences. ATD therapy is the first-line treatment for European and Asian patients (84-97\%), whereas RAI therapy is frequently chosen for American patients (59-75\%) [3-6]. As in other Asian countries, ATD is the most frequently selected treatment for GD in South Korea. Surveys conducted by the Korean Thyroid Association (KTA) demonstrated that $81 \%$ of respondents in 1991 and $97 \%$ in 2012 chose ATD as the first-line treatment for GD [7,8]. Data from the Korean Health Insurance Review and Assessment Service (HIRA; 2006-2012) consistently showed that 91-98\% of GD patients received ATD as the initial treatment [9]. Meanwhile, the initial remission rates for ATD therapy have been reported to 
be $40-50 \%$ even after 18 to 24 months of treatment $[10,11]$, suggesting that more than half of GD patients need second-line treatment. However, ablation therapies such as RAI ablation or thyroidectomy were conducted in fewer than $10 \%$ of GD patients in South Korea, which is a considerably lower proportion than that of patients who require second-line therapy [9]. Indeed, a previous study showed that a subset of patients received ATD therapy three or more times with repeated recurrence of GD [11].

This study aimed to investigate the clinical characteristics and the long-term outcomes of GD patients who underwent ablation therapy by evaluating 15 years of clinical experience at a single referral hospital.

\section{Materials and Methods}

\subsection{Subjects}

For this retrospective study, 10,991 adult patients (20 years old or older) who had been treated for GD at a single tertiary referral hospital between 2001 and 2015 were identified. GD was defined based on laboratory data including the level of the thyroid stimulating hormone (TSH) receptor antibody and/or increased diffuse thyroid uptake of ${ }^{99} \mathrm{~m}$ Tc on radionuclide scintigraphy. Patients who underwent ablation therapy for GD were screened $(n=1504)$, and those who had received initial ablation therapy before 2001 $(n=128)$ were excluded. Thus, 1357 patients were included in the final analysis. Data on age, sex, the time of diagnosis, the reason for ablation therapy, the presence of Graves' ophthalmopathy (GO), the pathologic results of surgery, and complications of surgery were obtained retrospectively through a review of patients' electronic medical records. The presence of GO was identified based on the International Classification of Diseases, 10th revision code H06.2, and/or details of a visit to an ophthalmologist in our hospital for GO. The severity of GO was divided into mild and moderate-to-severe GO based on the European group on Graves' Orbitopathy (EUGOGO) guideline [12]. The study was approved by the Institutional Review Board of Seoul National University Hospital (No. 1410-097-619).

\subsection{Definition of the Clinical Outcomes of GD}

Remission of GD was defined as the achievement of euthyroid or hypothyroid status following the withdrawal of ATD for more than 6 months. Immunologic remission was assessed by the achievement of the negative conversion of TSH receptor antibody after ablation therapy. TSH receptor antibody was measured using a radio-receptor assay kit (RSR Limited, Cardiff, UK) [11]. Permanent postoperative hypoparathyroidism was defined as persistent hypocalcemia with low parathyroid hormone levels $(<10 \mathrm{pg} / \mathrm{mL})$ for more than 1 year after surgery.

\subsection{Statistical Analysis}

Data are presented as the mean \pm standard deviation. The chi-square test was used to evaluate the changes in ablation therapy over time. The characteristics of patients who received RAI therapy and surgery were compared, and categorical and continuous variables were analyzed using the chi-square test and the Student $t$-test, respectively. To compare the cancer characteristics of incidentally found thyroid cancer after surgery and thyroid cancer suspected before surgery, categorical and continuous variables were analyzed using the chisquare test and the Mann-Whitney $U$ test, respectively. $p$ values $<0.05$ were considered to indicate statistical significance. All statistical analyses were performed using SPSS version 23.0 for Windows (IBM Corp., Armonk, NY, USA).

\section{Results}

\subsection{Trends in Ablation Therapy for GD during the 15-Year Period}

During the 15-year study period, a total of 10,991 patients were treated for GD, and $1357(12.3 \%)$ patients underwent ablation therapy, including RAI therapy or thyroidectomy. All patients received ATD therapy as the first-line treatment of GD, except five patients 
who initially received RAI therapy because of liver function abnormalities. Figure 1 shows the changes in the proportion of patients who underwent each type of ablation therapy for 15 years, divided into 5-year periods. The proportion of patients who underwent ablation therapy significantly decreased from 9.0\% (2001-2005) to 7.7\% (2011-2015) ( $p$ for trend $=0.03$; Figure 1a). The proportion of patients who underwent surgery significantly increased from $1.1 \%$ to $2.4 \%$, whereas the proportion of patients who received RAI therapy significantly decreased from $8.0 \%$ to $5.3 \%$ over time (all $p$ for trend $<0.01$; Figure 1a). During the 15-year period, the extent of surgery changed. The proportion of subtotal thyroidectomy decreased, as it was replaced by total thyroidectomy ( $p$ for trend $<0.01$; Figure 1b).
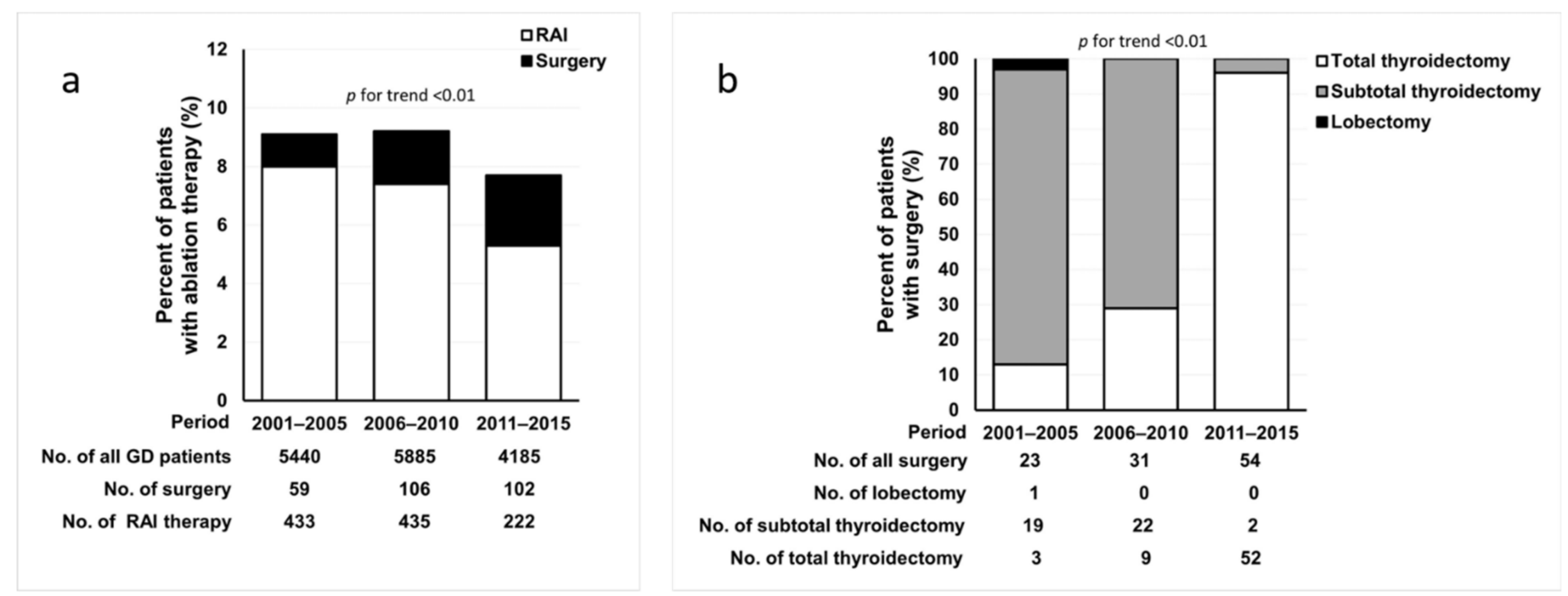

Figure 1. The trend of ablation therapy for Graves' disease (GD) over 15 years. (a) Changes in radioactive iodine (RAI) therapy and surgery. (b) Changes in the extent of surgery.

\subsection{Changes in the Reasons for Ablation Therapy for GD during the 15-Year Period}

Overall, the most common reason for receiving ablation therapy was intractable GD with or without large goiter (77\%), followed by adverse events of ATD therapy $(13 \%)$ and suspected thyroid cancer (10\%). During the 15-year period, the proportion of patients who received ablation therapy for suspected thyroid cancer increased significantly from $5.3 \%$ to $13.0 \%$ (Figure 2a), and all of those patients underwent surgery. The proportion of the patients who underwent surgery due to suspected thyroid cancer significantly increased in the 2006-2010 period and subsequently decreased in the 2011-2015 period (Figure 2b). Meanwhile, the reasons for RAI therapy did not change over time (Figure 2c).

During the 15-year period, 10,986 GD patients initially received ATD treatment, and 154 patients $(1.4 \%)$ then required ablation therapy due to severe adverse events. The proportion of these patients decreased from 1.1\% (2001-2005) and 1.2\% (2006-2010) to $0.7 \%$ (2011-2015) ( $p$ for trend $=0.047)$. The most common adverse event was toxic hepatitis $(47 \%)$, followed by agranulocytosis (31\%) and urticaria and/or skin rash (23\%) (Supplementary Table S1). The incidence of toxic hepatitis and urticaria and/or skin rash was significantly higher in propylthiouracil (PTU) users than in methimazole users. RAI therapy was preferred over surgery in these patients $(96 \%$ vs. $4 \%, p<0.01)$. 

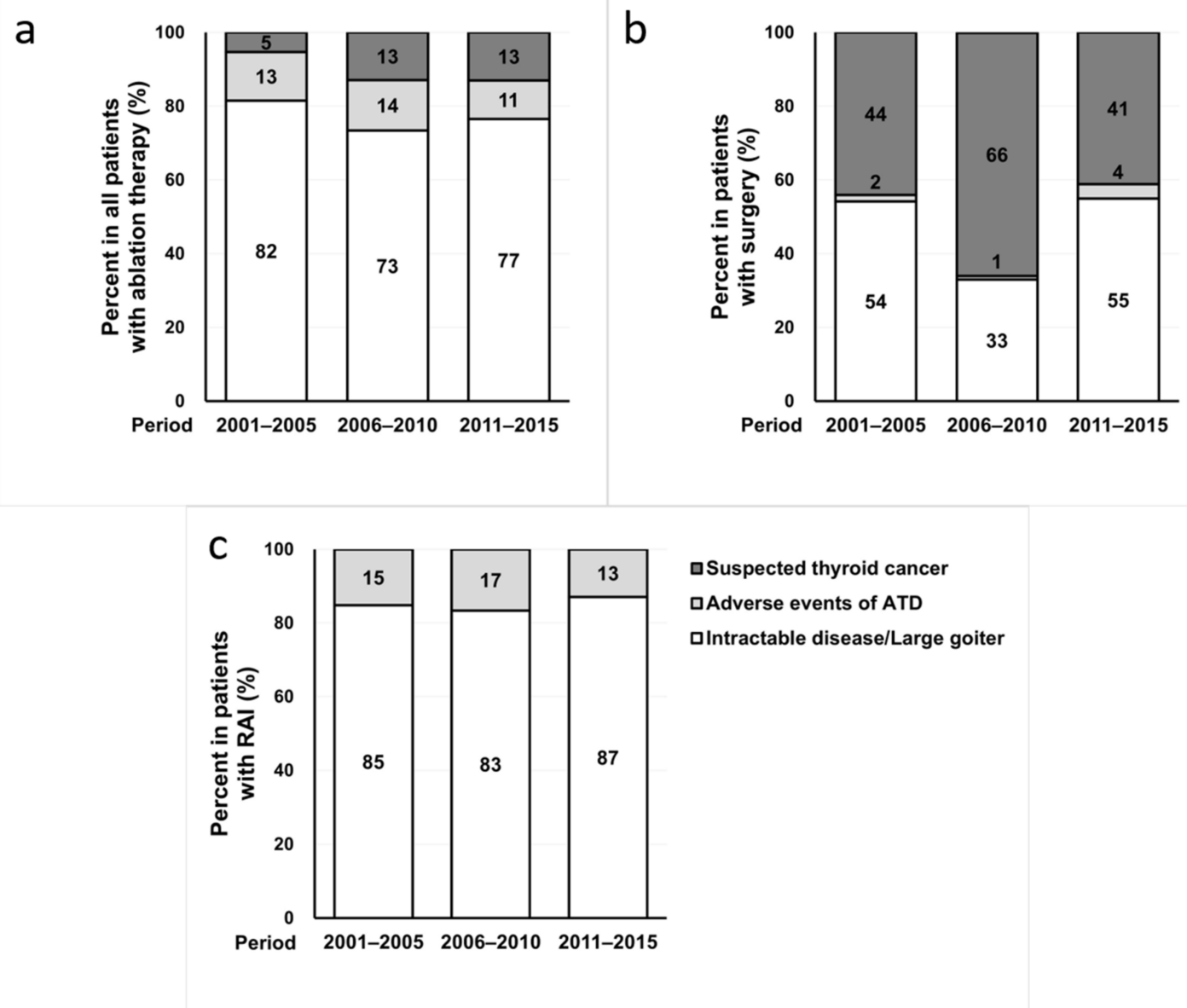

Figure 2. Changes in the reason for ablation therapy for Graves' disease over time. (a) All patients, $n=10,991$. (b) Patients who received surgery, $n=267$. (c) Patients who received RAI therapy, $n=1090$.

\subsection{Changes in the Clinical Characteristics of Patients during the 15-Year Period}

Among the 1357 patients who underwent ablation therapy for GD, patients with a follow-up duration of less than 1 year $(n=156)$ were excluded, and the clinical characteristics of the remaining patients were analyzed. The mean age of the study subjects was $42 \pm 13$ years, and $71 \%$ were women. The time from diagnosis to ablation therapy was $5.1 \pm 5.2$ years. Over 15 years, the age at ablation therapy and the time from diagnosis to ablation therapy significantly increased (Table 1 ). The proportion of women of childbearing age (aged 20-44) significantly decreased to 47\% (2001-2005), 40\% (2006-2010), and 36\% $(2011-2015)$ ( $p$ for trend $=0.012)$. Although the prevalence of GO increased over time, the prevalence of moderate-to-severe GO did not differ (Table 1).

Table 1. Comparison of clinical characteristics for patients who underwent ablation therapy according to the period.

\begin{tabular}{|c|c|c|c|c|c|}
\hline Characteristics & $\begin{array}{c}\text { Total } \\
(n=1201)\end{array}$ & $\begin{array}{c}2001-2005 \\
(n=432)\end{array}$ & $\begin{array}{c}2006-2010 \\
(n=474)\end{array}$ & $\begin{array}{c}2011-2015 \\
(n=295)\end{array}$ & $\begin{array}{c}p \\
\text { for trend }\end{array}$ \\
\hline Age at ablation therapy (years) & $42 \pm 13$ & $39 \pm 12$ & $42 \pm 13$ & $46 \pm 14$ & $<0.001$ \\
\hline Sex, female, $n(\%)$ & $855(71)$ & $309(72)$ & $322(68)$ & $224(76)$ & 0.300 \\
\hline Time from diagnosis to ablation therapy (years) & $5.1 \pm 5.2$ & $4.4 \pm 4.8$ & $4.6 \pm 4.8$ & $6.6 \pm 5.9$ & $<0.001$ \\
\hline $\mathrm{GO}, n(\%)$ & $89(7)$ & $17(4)$ & $43(9)$ & $29(10)$ & 0.002 \\
\hline Moderate-to-severe GO, $n(\%)$ & $16(1.3)$ & $5(1.2)$ & $7(1.5)$ & $4(1.4)$ & 0.915 \\
\hline
\end{tabular}

Data were presented as mean \pm standard deviation or $n(\%)$. GO, Graves' ophthalmopathy. 


\subsection{Clinical Characteristics of Patients According to the Treatment Modality}

Next, the clinical characteristics of patients were analyzed according to treatment modality (RAI vs. surgery), and the surgery group was further divided according to the reason for surgery (the surgery for GD therapy, designated "surgery for GD", and surgery for suspected cancer, designated "surgery for cancer").

First, the RAI and surgery for GD groups were compared, since the reason for ablation therapy was similar in these two groups. The surgery for GD group showed a higher ratio of female sex ( $86 \%$ vs. $69 \%, p<0.01)$, while the age at ablation therapy was similar between the two groups (Table 2). The proportion of women of childbearing age was significantly lower in the surgery for GD group than that in the RAI group (46\% vs. 59\%, $p=0.01$ ). The time from diagnosis to ablation therapy in the surgery for GD group was significantly longer than that in the RAI group $(6.9 \pm 6.9$ vs. $4.9 \pm 4.8$ years, $p<0.01)$. The prevalence of moderate-to-severe GO in the surgery for GD group was significantly higher than that in the RAI group ( $3.9 \%$ vs. $1.2 \%, p=0.04)$. Among 12 patients with moderate-to-severe GO in the RAI group, seven patients with active GO received steroid treatment to prevent the worsening GO. A higher proportion of patients underwent ablation therapy due to adverse events of ATD in the RAI group than in the surgery for GD group (15\% vs. $6 \%, p<0.01$; Table 2).

Table 2. Comparison of clinical characteristics among RAI and surgery groups.

\begin{tabular}{|c|c|c|c|}
\hline Characteristics & $\begin{array}{c}\text { RAI } \\
(n=968)\end{array}$ & $\begin{array}{l}\text { Surgery for GD } \\
\quad(n=106)\end{array}$ & $\begin{array}{l}\text { Surgery for Cancer } \\
\quad(n=127)\end{array}$ \\
\hline Age at ablation therapy (years) & $41 \pm 13$ & $39 \pm 14$ & $48 \pm 14^{\mathrm{a}, \mathrm{b}}$ \\
\hline Sex, female, $n(\%)$ & $663(69)$ & $91(86)^{\mathrm{a}}$ & $101(80)^{\mathrm{a}}$ \\
\hline Time from diagnosis to ablation therapy (years) & $4.9 \pm 4.8$ & $6.9 \pm 6.9^{a}$ & $4.4 \pm 6.0$ \\
\hline Follow-up duration (years) & $7.2 \pm 4.4$ & $5.5 \pm 3.3^{a}$ & $7.2 \pm 3.1^{b}$ \\
\hline $\mathrm{GO}, n(\%)$ & $67(7)$ & $10(9)$ & $12(9)$ \\
\hline Moderate-to-severe GO, $n(\%)$ & $12(1.2)$ & $4(3.8)^{\mathrm{a}}$ & $0(0)$ \\
\hline \multicolumn{4}{|l|}{ Reason for ablation therapy, $n(\%)$} \\
\hline Intractable disease \pm large goiter & $820(85)$ & $100(94)^{\mathrm{a}}$ & \\
\hline Adverse events of ATD & $148(15)$ & $6(6)^{a}$ & \\
\hline Suspected thyroid cancer & & & $127(100)^{a, b}$ \\
\hline
\end{tabular}

Data were presented as mean \pm standard deviation or $n$ (\%). RAI, radioactive iodine; GO, Graves' ophthalmopathy; ATD, anti-thyroid drug. ${ }^{\mathrm{a}} p<0.05$ compared to the RAI group; ${ }^{\mathrm{b}} p<0.05$ compared to the surgery for GD.

Next, the patients who underwent surgery for cancer were analyzed. Patients' age at ablation therapy was older than that of the other two groups, and the female ratio was higher than that of the RAI group but similar to that of the surgery for GD group (Table 2). The time from diagnosis to ablation therapy and the follow-up duration were similar to those of the RAI group (Table 2).

\subsection{Clinical Outcomes of Ablation Therapy}

After a median follow-up duration of 6.2 years (range, 1.0-17.7 years), 86\% (835/968) and $98 \%(229 / 233)$ of patients in the RAI and surgery groups, respectively, achieved remission. The remission rates for RAI therapy and surgery were similar during the three consecutive 5-year periods (Supplementary Figure S1). As expected, surgery achieved a significantly higher remission rate $(p<0.01)$ despite a shorter follow-up duration (Table 2). After ablation therapy, 995 (83\%) patients measured TSH receptor antibody at least once. Among 869 patients who achieved final remission, 390 (45\%) patients have been proved to obtain the immunological remission, achieving negative conversion of TSH receptor antibody measurement.

In the surgery group, four patients experienced recurrence. All of them received subtotal thyroidectomy, and their mean time from surgery to recurrence was 6.3 years (range, 3.1-8.9 years). Meanwhile, there was no recurrence in patients who underwent 
total thyroidectomy. Serious surgical complications, including postoperative permanent hypoparathyroidism and vocal cord palsy, developed in $10(4.3 \%)$ and two $(0.9 \%)$ patients, respectively, and the rate of complications did not significantly differ according to whether patients underwent subtotal thyroidectomy or total thyroidectomy ( $4 \%$ vs. $8 \%, p=0.26$ ).

Postoperative pathological outcomes were also analyzed. Among the 127 patients in the surgery for cancer group, $110(87 \%)$ had pathologically confirmed thyroid cancer, and $96 \%$ of 110 patients had differentiated thyroid cancer. Interestingly, $10 \%(11 / 106)$ of the patients in the surgery for GD group had incidentally found thyroid cancer after surgery. These incidentally found thyroid cancers were smaller $(0.7 \pm 0.7$ vs. $1.3 \pm 1.2 \mathrm{~cm}, p<0.01)$ and had fewer extrathyroidal extensions ( $9 \%$ vs. $50 \%, p=0.01$ ) than those of the thyroid cancers suspected before surgery (Table 3).

Table 3. Comparison of cancer characteristics between incidentally found thyroid cancer after surgery and thyroid cancer suspected before surgery.

\begin{tabular}{|c|c|c|c|}
\hline Characteristics & $\begin{array}{c}\text { Incidental Cancer } \\
\text { (Surgery for GD) } \\
(n=11)\end{array}$ & $\begin{array}{c}\text { Suspected Cancer } \\
\text { (Surgery for Cancer) } \\
(n=110)\end{array}$ & $p$ \\
\hline Mean age at surgery (years) & $42 \pm 13$ & $48 \pm 13$ & 0.19 \\
\hline$<55$ years & $9(82)$ & $74(67)$ & 0.50 \\
\hline$\geq 55$ years & $2(18)$ & $36(33)$ & \\
\hline Sex, female, $n(\%)$ & $10(91)$ & $86(78)$ & 0.46 \\
\hline \multicolumn{4}{|l|}{ Tumor type, $n(\%)$} \\
\hline PTC & $9(82)$ & $97(88)$ & 0.46 \\
\hline FTC & $2(18)$ & $9(8)$ & \\
\hline PDTC & $0(0)$ & $2(2)$ & \\
\hline MTC & $0(0)$ & $2(2)$ & \\
\hline Tumor size $(\mathrm{cm}), n(\%)$ & $0.7 \pm 0.7$ & $1.3 \pm 1.2$ & $<0.01$ \\
\hline$\leq 0.5 \mathrm{~cm}$ & $8(73)$ & $25(23)$ & $<0.01$ \\
\hline $0.6-1.0 \mathrm{~cm}$ & $1(9)$ & $39(35)$ & \\
\hline $1.1-2.0 \mathrm{~cm}$ & $1(9)$ & $29(26)$ & \\
\hline$>2.0 \mathrm{~cm}$ & $1(9)$ & $17(16)$ & \\
\hline Multiplicity, $n(\%)$ & $3(27)$ & $40(36)$ & 0.75 \\
\hline ETE, $n(\%)$ & $1(9)$ & $54(50)$ & 0.01 \\
\hline LN metastasis, $n(\%)$ & $1(9)$ & $25(23)$ & 0.45 \\
\hline
\end{tabular}

Data were presented as mean \pm standard deviation or $n(\%)$. PTC, papillary thyroid carcinoma; FTC, follicular thyroid carcinoma; PDTC, poorly differentiated thyroid carcinoma; MTC, medullary thyroid carcinoma; ETE, extra-thyroidal extension; LN, lymph node.

\section{Discussion}

Among 10,991 GD patients, 1357 (12.3\%) received ablation therapy, including RAI therapy or surgery, as the second-line treatment of GD. The most common reason for ablation therapy was intractable disease with or without a large goiter, followed by adverse events of ATD and suspicion of thyroid cancer. The proportion of GD patients who underwent RAI therapy significantly decreased during the 15-year period, whereas the proportion of patients who underwent surgery significantly increased in association with the increased rate of suspected thyroid cancer. After a median 6.2-year follow-up duration, the remission rate was significantly higher in the surgery group than in the RAI group, whereas both remission rates were markedly higher (86-98\%) than in patients who received ATD therapy (52\%), in accordance with the findings of a previous study [11].

During the 15-year period, the proportion of patients who underwent surgery within the ablation therapy group and the proportion of patients who underwent surgery for suspected thyroid cancer significantly increased. Compared to 2001-2005, the diagnosis of thyroid cancer increased in 2006-2015 with the technological evolution of high-resolution ultrasonography test in Korea [13,14]. Therefore, the proportion of RAI therapy has decreased relatively. An emerging question is whether cancer screening should be performed before selecting the treatment modality for GD patients. At present, clinical practice 
guidelines recommend no further cancer screening for all GD patients; however, cancer screening is performed with considerable frequency in real-world clinical practice. This study showed that $10 \%$ of the patients in the surgery for GD group had incidentally diagnosed thyroid cancers. Previous studies have reported that the pooled prevalence of incidental thyroid cancer was 7\% (0.4-32.4\%) [15]. Although $73 \%$ of these tumors were smaller than $0.5 \mathrm{~cm}$, similar to the findings from autopsy data [16,17], $27 \%$ and $9 \%$ of them had multiplicity and lymph node metastasis, respectively. Thus, careful examinations may be needed when preparing for surgery. However, routine cancer screening for all GD patients is unfeasible due to the heterogeneous parenchymal changes in ultrasonographic imaging of these patients. In addition, the prevalence of thyroid cancer in GD patients has been reported to be similar to that in patients with euthyroid goiter or hyperthyroid patients without GD $[15,18]$. Moreover, the incidentally found small thyroid cancers in GD patients have shown a better prognosis than those found in the general population [19]. Therefore, further evidence is needed to conclusively answer this question.

This study demonstrated that ablation therapy was required in at least $12 \%$ of patients with GD after initial ATD treatment. In real-world practice, the selection of an optimal ablation therapy—-that is, the choice between RAI therapy and surgery—is difficult except for patients with suspected thyroid cancer (surgery for cancer group). Although a longterm follow-up study of quality of life revealed no significant differences between RAI therapy and surgery [20], treatment should be carefully chosen in consideration of several factors, such as age, sex, goiter size, the presence of GO, comorbidities, and the individual patient's socio-economic status. In this study, the surgery for GD group showed a higher proportion of female patients and a longer time from diagnosis to ablation therapy than the RAI group. However, the other characteristics did not differ significantly between the two groups. The presence of GO is an important factor in decision making. Because RAI therapy can induce and worsen GO [21], surgery is recommended over RAI therapy. Previous studies have reported that GO was associated with the decision to choose surgery over RAI therapy [22]. In this study, the prevalence of moderate-to-severe GO was also significantly higher in the surgery group than in the RAI group (3.9\% vs. $1.2 \%)$, although the total prevalence of GO including mild form did not differ between groups $(9.4 \%$ vs. $6.9 \%$ ). The possible reason for the similar prevalence of GO may be that RAI therapy can be safely performed with steroid use in mild cases of GO [1,12]. Too low prevalence of GO may be another reason for the limited difference between groups. GO is less frequent and less severe in Asians than in Caucasians [23,24]. In addition, the presence of GO, especially mild cases, may have been undervalued and missed because it identified based on the diagnosis code and/or ophthalmology visit record in this study. In this study, patients with GO increased from $4 \%$ (2001-2005) to $10 \%$ (2011-2015), but the prevalence of moderate-to-severe GO did not differ over 15 years. In the 2010s, the rapid referral system between the thyroid center and ophthalmology team has been established in our hospital; thus, doctors became more interested in GO and referred to the ophthalmologist. Therefore, the increase in the prevalence of recorded GO reflects the increase in diagnosis of mild GO owing to the increase in doctor's interest of it.

Of the patients who underwent ablation therapy, $13 \%$ underwent these treatments because of adverse events of ATD, similar to the proportion in previous reports [3]. Methimazole is the preferred ATD because of its effectiveness and favorable adverse event profile [6], and the use of methimazole has been increasing compared to that of PTU [3,9]; methimazole accounted for $55 \%$ in Korea and $60 \%$ in the United States, respectively [3,9]. Because methimazole is used more often, adverse events of methimazole are more frequently reported (Supplementary Table S1). Among the adverse events, toxic hepatitis was the most common cause for ablation therapy.

The remission rate of thyroidectomy $(98 \%)$ was remarkably higher than that of RAI therapy $(86 \%)$. A systematic review of 62 studies reported that thyroidectomy is more likely to be successful than RAI therapy [25]. In terms of surgical extent, the recurrence rate for total thyroidectomy is significantly lower than that for subtotal thyroidectomy, with 
an odds ratio of 0.1 [26,27]. In Korea, Sung et al. reported that the recurrence rate of GD was lower in patients who underwent total thyroidectomy than in those who underwent subtotal thyroidectomy ( $0 \%$ vs. $4.1 \%$ ) [28], which is similar to the results of this study. However, the higher surgical complication rate of total thyroidectomy than that of subtotal thyroidectomy is an obstacle in choosing this procedure [26]. However, a meta-analysis of randomized clinical trials showed that the rate of permanent hypoparathyroidism and recurrent laryngeal nerve palsy after total thyroidectomy was not significantly different from that after subtotal thyroidectomy [27]. Furthermore, this study showed that total thyroidectomy had a significantly lower recurrence rate without increasing the rate of surgical complications. Therefore, total thyroidectomy is the recommended procedure of choice [1], and the preference for the surgical procedure has shifted from subtotal to total thyroidectomy over time (Figure 1b) [29].

Ablation therapy is indispensable for GD treatment because initial ATD treatment cannot achieve remission in all patients. Ablation therapy was performed in $12 \%$ of patients over 15 years. Although it is difficult to choose between RAI therapy and surgery, both types of ablation therapy showed satisfactory remission rates. Therefore, ablation therapy is actively recommended in patients who do not achieve remission after ATD therapy.

Supplementary Materials: The following are available online at https://www.mdpi.com/article/10 $.3390 /$ jcm10081629/s1, Table S1: Adverse effects of ATD requiring the ablation therapy, Figure S1: Remission rate for RAI therapy and surgery over time.

Author Contributions: Conceptualization, M.J.K., Y.A.K., S.W.C., and Y.J.P.; data curation and formal analysis, M.J.K., Y.A.K., S.-j.K., K.E.L., Y.J.P., D.J.P., and B.Y.C.; writing-original draft preparation, M.J.K.; writing-review and editing, M.J.K., Y.A.K., S.W.C., and Y.J.P.; funding acquisition, S.W.C. All authors have read and agreed to the published version of the manuscript.

Funding: This research was funded by the National Research Foundation of Korea (NRF) grant funded by the Korea government (NRF-2019R1A2C1085672).

Institutional Review Board Statement: The study was conducted according to the guidelines of the Declaration of Helsinki and approved by the Institutional Review Board of Seoul National University Hospital (No. 1410-097-619).

Informed Consent Statement: Patient consent was waived due to the retrospective design of the study.

Data Availability Statement: Data sharing not applicable.

Acknowledgments: The Biospecimens and data used in this study were provided by the Biobank of Seoul National University Hospital, a member of Korea Biobank Network.

Conflicts of Interest: The authors declare no conflict of interest.

\section{References}

1. Ross, D.S.; Burch, H.B.; Cooper, D.S.; Greenlee, M.C.; Laurberg, P.; Maia, A.L.; Rivkees, S.A.; Samuels, M.; Sosa, J.A.; Stan, M.N. American Thyroid Association Guidelines for Diagnosis and Management of Hyperthyroidism and Other Causes of Thyrotoxicosis. Thyroid 2016, 26, 1343-1421. [CrossRef] [PubMed]

2. Yi, K.H.; Moon, J.H.; Kim, I.; Bom, H.; Lee, J.; Chung, W.Y.; Chuang, J.H.; Shong, Y.K. The Diagnosis and Management of Hyperthyroidism Consensus-Report of the Korean Thyroid Association. J. Korean Thyroid Assoc. 2013, 6, 1-11. [CrossRef]

3. Sundaresh, V.; Brito, J.P.; Thapa, P.; Bahn, R.S.; Stan, M.N. Comparative Effectiveness of Treatment Choices for Graves' Hyperthyroidism: A Historical Cohort Study. Thyroid 2017, 27, 497-505. [CrossRef] [PubMed]

4. Kornelius, E.; Yang, Y.S.; Huang, C.N.; Wang, Y.H.; Lo, S.C.; Lai, Y.R.; Chiou, J.-Y. The Trends of Hyperthyroidism Treatment in Taiwan: A Nationwide Population-Based Study. Endocr. Pract. 2018, 24, 573-579. [CrossRef]

5. Bartalena, L.; Burch, H.B.; Burman, K.D.; Kahaly, G.J. A 2013 European survey of clinical practice patterns in the management of Graves' disease. Clin. Endocrinol. 2016, 84, 115-120. [CrossRef]

6. Burch, H.B.; Burman, K.D.; Cooper, D.S. A 2011 survey of clinical practice patterns in the management of Graves' disease. J. Clin. Endocrinol. Metab. 2012, 97, 4549-4558. [CrossRef]

7. Cho, B.Y.; Koh, C.S. Current Trends in The Diagnosis and Treatment of Graves' Disease in Korea. J. Korean Soc. Endocrinol. 1992, 7, 216-227. 
8. Moon, J.H.; Yi, K.H. The diagnosis and management of hyperthyroidism in Korea: Consensus report of the korean thyroid association. Endocrinol. Metab. 2013, 28, 275-279. [CrossRef]

9. Seo, G.H.; Kim, S.W.; Chung, J.H. Incidence \& Prevalence of Hyperthyroidism and Preference for Therapeutic Modalities in Korea. J. Korean Thyroid Assoc. 2013, 6, 56.

10. Sundaresh, V.; Brito, J.P.; Wang, Z.; Prokop, L.J.; Stan, M.N.; Murad, M.H.; Bahn, R.S. Comparative effectiveness of therapies for Graves' hyperthyroidism: A systematic review and network meta-analysis. J. Clin. Endocrinol. Metab. 2013, 98, 3671-3677. [CrossRef]

11. Kim, Y.A.; Cho, S.W.; Choi, H.S.; Moon, S.; Moon, J.H.; Kim, K.W.; Park, D.J.; Yi, K.H.; Park, Y.J.; Cho, B.Y. The Second Antithyroid Drug Treatment Is Effective in Relapsed Graves' Disease Patients: A Median 11-Year Follow-Up Study. Thyroid 2017, 27, 491-496. [CrossRef]

12. Bartalena, L.; Baldeschi, L.; Boboridis, K.; Eckstein, A.; Kahaly, G.J.; Marcocci, C.; Perros, P.; Salvi, M.; Wiersinga, W.M. The 2016 European Thyroid Association/European Group on Graves' Orbitopathy Guidelines for the Management of Graves' Orbitopathy. Eur. Thyroid. J. 2016, 5, 9-26. [CrossRef]

13. Moon, J.H.; Hyun, M.K.; Lee, J.Y.; Shim, J.I.; Kim, T.H.; Choi, H.S.; Ahn, H.Y.; Kim, K.W.; Park, D.J.; Park, Y.J.; et al. Prevalence of thyroid nodules and their associated clinical parameters: A large-scale, multicenter-based health checkup study. Korean J. Intern. Med. 2018, 33, 753-762. [CrossRef]

14. Yi, K.H.; Lee, E.K.; Kang, H.-C.; Koh, Y.; Kim, S.W.; Kim, I.J.; Na, D.G.; Ryu, J.-S.; Park, S.Y.; Park, I.A.; et al. Revised Korean Thyroid Association Management Guidelines for Patients with Thyroid Nodules and Thyroid Cancer. Int. J. Thyroidol. 2016, 9, 59. [CrossRef]

15. Jia, Q.; Li, X.; Liu, Y.; Li, L.; Kwong, J.S.; Ren, K.; Jiang, Y.; Sun, X.; Tian, H.; Li, S. Incidental thyroid carcinoma in surgery-treated hyperthyroid patients with Graves' disease: A systematic review and meta-analysis of cohort studies. Cancer Manag. Res. 2018, 10, 1201-1207. [CrossRef]

16. Lee, Y.S.; Lim, H.; Chang, H.S.; Park, C.S. Papillary thyroid microcarcinomas are different from latent papillary thyroid carcinomas at autopsy. J. Korean Med. Sci. 2014, 29, 676-679. [CrossRef]

17. Leboulleux, S.; Tuttle, R.M.; Pacini, F.; Schlumberger, M. Papillary thyroid microcarcinoma: Time to shift from surgery to active surveillance? Lancet Diabetes Endocrinol. 2016, 4, 933-942. [CrossRef]

18. Ergin, A.B.; Saralaya, S.; Olansky, L. Incidental papillary thyroid carcinoma: Clinical characteristics and prognostic factors among patients with Graves' disease and euthyroid goiter, Cleveland Clinic experience. Am. J. Otolaryngol. 2014, 35, 784-790. [CrossRef]

19. Kikuchi, S.; Noguchi, S.; Yamashita, H.; Uchino, S.; Kawamoto, H. Prognosis of small thyroid cancer in patients with Graves' disease. Br. J. Surg. 2006, 93, 434-439. [CrossRef]

20. Abraham-Nordling, M.; Torring, O.; Hamberger, B.; Lundell, G.; Tallstedt, L.; Calissendorff, J. Graves' disease: A long-term quality-of-life follow up of patients randomized to treatment with antithyroid drugs, radioiodine, or surgery. Thyroid 2005, 15, 1279-1286. [CrossRef]

21. Li, H.X.; Xiang, N.; Hu, W.K.; Jiao, X.L. Relation between therapy options for Graves' disease and the course of Graves' ophthalmopathy: A systematic review and meta-analysis. J. Endocrinol. Investig. 2016, 39, 1225-1233. [CrossRef] [PubMed]

22. Elfenbein, D.M.; Schneider, D.F.; Havlena, J.; Chen, H.; Sippel, R.S. Clinical and socioeconomic factors influence treatment decisions in Graves' disease. Ann. Surg. Oncol. 2015, 22, 1196-1199. [CrossRef] [PubMed]

23. Tellez, M.; Cooper, J.; Edmonds, C. Graves' ophthalmopathy in relation to cigarette smoking and ethnic origin. Clin. Endocrinol. 1992, 36, 291-294. [CrossRef] [PubMed]

24. Khong, J.J.; Finch, S.; De Silva, C.; Rylander, S.; Craig, J.E.; Selva, D.; Ebeling, P.R. Risk Factors for Graves' Orbitopathy; the Australian Thyroid-Associated Orbitopathy Research (ATOR) Study. J. Clin. Endocrinol. Metab. 2016, 101, 2711-2720. [CrossRef]

25. Genovese, B.M.; Noureldine, S.I.; Gleeson, E.M.; Tufano, R.P.; Kandil, E. What is the best definitive treatment for Graves' disease? A systematic review of the existing literature. Ann. Surg. Oncol. 2013, 20, 660-667. [CrossRef]

26. Feroci, F.; Rettori, M.; Borrelli, A.; Coppola, A.; Castagnoli, A.; Perigli, G.; Cianchi, F.; Scatizzi, M. A systematic review and meta-analysis of total thyroidectomy versus bilateral subtotal thyroidectomy for Graves' disease. Surgery 2014, 155, 529-540. [CrossRef]

27. Guo, Z.; Yu, P.; Liu, Z.; Si, Y.; Jin, M. Total thyroidectomy vs bilateral subtotal thyroidectomy in patients with Graves' diseases: A meta-analysis of randomized clinical trials. Clin. Endocrinol. 2013, 79, 739-746.

28. Sung, T.Y.; Kim, Y.S.; Lee, S.H.; Yoon, J.H.; Hong, S.J. Surgical Treatment of Graves' Disease: Comparison between Total Thyroidectomy and Subtotal Thyroidectomy. J. Korean Surg. Soc. 2009, 77, 82-87. [CrossRef]

29. Sung, T.Y.; Lee, Y.M.; Yoon, J.H.; Chung, K.W.; Hong, S.J. Long-Term Effect of Surgery in Graves' Disease: 20 Years Experience in a Single Institution. Int. J. Endocrinol. 2015, 2015, 542641. [CrossRef] 